\title{
Cytomegalovirus-associated pulmonary exacerbation in patients with cystic fibrosis
}

To the Editor:

Cystic fibrosis (CF) is a multisystem disease characterised by recurrent respiratory tract infections and progressive respiratory failure. It remains one of the most common life-threatening autosomal recessive conditions affecting Caucasians. Recent studies have highlighted the association between respiratory viral infections and frequency of pulmonary exacerbations, increase antibiotic usage and poorer outcome $[1,2]$.

Cytomegalovirus (CMV) is a DNA virus and a member of the herpes family of viruses, which includes herpes simplex virus, Epstein-Barr virus (EBV) and varicella-zoster virus. Like these other viruses, once primary infection has occurred, CMV establishes itself in the host in a latent form with periodic episodes of reactivation occurring throughout life. CMV is a common infection with most individuals acquiring infection at some time. Both primary and nonprimary infections are associated with viral shedding in urine, saliva, semen, cervical secretions, breast milk and other body fluids. In healthy individuals, symptoms of CMV infection are often mild and nonspecific, or absent altogether, and rarely cause serious illness. However, CMV infection can be life threatening to immunocompromised individuals, such as those with advanced HIV infection, transplant recipients or very premature infants.

We describe three cases of primary CMV infection causing significant pulmonary exacerbation in adults with CF.

The first case involved a 20-year-old woman with CF who presented with a 1-week history of dyspnoea and productive cough with green sputum. She complained of generalised headaches, tinnitus, nausea, vomiting and loose stools. She had CF-related diabetes (CFRD) and was chronically colonised with Pseudomonas aeruginosa. There was no history of sore throat, rash or fever. Lung function had deteriorated, full blood count (FBC) was unremarkable and C-reactive protein (CRP) was elevated at $51 \mathrm{mg} \cdot \mathrm{L}^{-1}$. A viral throat swab was negative, as was the EBV monospot. A high-resolution computed tomography scan demonstrated widespread bronchiectasis with no focal consolidation. Other investigations included a negative CMV PCR and blood cultures. Despite broad-spectrum antibiotics, she remained dyspnoeic, had persistent high, fluctuating temperatures, and lung function remained unchanged. Sputum cultures were positive for $P$. aeruginosa, which was fully sensitive to her antibiotic regimen, CRP remained elevated at $45 \mathrm{mg} \cdot \mathrm{L}^{-1}$ and she developed an acute lymphocytosis of $11.53 \times 10^{9}$ per $\mathrm{L}$. A transthoracic echocardiography demonstrated moderately impaired left ventricular systolic function. A CMV IgM and IgG antibody test was positive with a PCR viral load of 286500 copies $\cdot \mathrm{mL}^{-1}$. A diagnosis of acute CMV infection was made and she was started on a 3 -week course of valganciclovir. There was a dramatic improvement in lung function and weight, and both fever and lymphocytosis resolved. A follow up echocardiography demonstrated mildly hypokinetic basal-to-midseptal contraction.

The second case occurred in a 21-year-old woman with CF and CFRD. She presented with a 3-week history of productive cough, chest tightness, spiking temperatures, fatigue, and a blanching maculopapular rash affecting her upper limbs and trunk. There was no lymphadenopathy or organomegaly. Forced expiratory volume in $1 \mathrm{~s}$ (FEV1) was significantly reduced at 56\% predicted from a baseline of $70-80 \%$ predicted. Blood lymphocyte count and CRP were elevated at $16.98 \times 10^{9}$ per L and $44 \mathrm{mg} \cdot \mathrm{L}^{-1}$, respectively.

@ERSpublications

CMV is an unusual cause of pulmonary exacerbation in immunocompetent individuals with $\mathrm{CF}$ http://ow.ly/Rdds30hlnjV

Cite this article as: Sawant A, Spoletini G, Whitaker P, et al. Cytomegalovirus-associated pulmonary exacerbation in patients with cystic fibrosis. ERJ Open Res 2018; 4: 00111-2017 [https:// doi.org/10.1183/23120541.00111-2017]. 
A chest radiograph showed patchy consolidation in the right mid- and upper zones, and blood cultures were negative. CMV IgM and IgG antibody test was positive with a PCR viral load of $23420 \mathrm{copies} \cdot \mathrm{mL}^{-1}$. A viral throat swab was negative, as was the EBV monospot. However, EBV PCR was positive with 961 copies $\cdot \mathrm{mL}^{-1}$. After 2 weeks of intravenous antibiotics she remained hyperglycaemic despite the introduction of insulin, and lung function remained low. Following a 2-week course of valganciclovir, she made a full recovery with good glycaemic control and an FEV1 of 71\% predicted. The CMV PCR titre fell to 1413 copies $\cdot \mathrm{mL}^{-1}$ and lymphocytosis resolved.

The final case involved a 20-year-old woman with CF who presented with high spiking temperatures, productive cough and general malaise. Sputum was chronically colonised with $P$. aeruginosa. On admission, FBC was unremarkable (white cell count $9.17 \times 10^{9}$ per L, lymphocyte count $1.44 \times 10^{9}$ per L), CRP was elevated at $122 \mathrm{mg} \cdot \mathrm{L}^{-1}$ and there was patchy consolidation on chest radiography. She had a $3-\mathrm{kg}$ weight loss and her FEV1 had dropped from $80 \%$ to $44 \%$ predicted. Viral throat swab PCR was positive for parainfluenza type 4. Despite a week of i.v. tobramycin, fosfomycin and oral clarithromycin, she continued to experience spikes in body temperature. Multiple peripheral and port cultures were negative as was a repeat viral throat swab but a repeat $\mathrm{FBC}$ showed acute lymphocytosis $\left(5.82 \times 10^{9}\right.$ per $\left.\mathrm{L}\right)$. In view of ongoing temperatures and lymphocytosis, a CMV/EBV PCR screen was performed. CMV PCR was positive with 83660 copies $\cdot \mathrm{mL}^{-1}$ whilst EBV and adenovirus were not detected. CMV IgM was positive and CMV IgG avidity was low, confirming active primary CMV infection. After 4 weeks of i.v. antibiotics, she made a slow recovery with symptom resolution. Her FEV 1 increasing to $74 \%$ predicted, lymphocytosis resolved and a repeat CMV PCR fell to 2432 copies $\cdot \mathrm{mL}^{-1}$.

CMV infection is an uncommon cause of respiratory complications in immunocompetent patients, although CMV pneumonia is being recognised more frequently in immunocompetent adults and children [3-6]. It is a leading cause of morbidity and mortality in immunosuppressed patients as well as very premature and congenitally infected infants. In lung transplant recipients, it is associated with acute syndromes and graft rejection $[7,8]$. There are no data on the prevalence and impact of CMV in patients with CF. A small study by ONG et al. [9], investigating serological evidence of CMV and respiratory viral infections in 36 patients with CF, reported a single case of CMV seroconversion in 11 patients with viral or mycoplasma infections. A further two cases of presumed CMV have been reported in abstract form [10]. Both cases were complicated by allergic bronchopulmonary aspergillosis and despite clinical features being consistent with CMV infection, there was no reference to CMV serology or PCR diagnosis.

The presentation in the three cases reported here was remarkably similar, although in case one seroconversion had not yet occurred and was only diagnosed after failure to respond to broad-spectrum antibiotics. The characteristic features were lymphocytosis, intermittent fever unresponsive to i.v. antibiotics and a persistent decline in lung function. In our unit, viral throat swabs are routinely taken in all patients presenting with acute pulmonary exacerbations and lymphocytosis is not associated with respiratory viral infections such as respiratory syncytial virus, influenza and parainfluenza. We have seen a similar case presentation in a patient with acute EBV infection although this was associated with significant lymphadenopathy. The diagnosis of primary CMV infection was based on the presence of CMV IgM and CMV PCR. All three patients had significant viral loads and low CMV IgG avidity. The two patients with the highest lymphocyte counts were treated with valganciclovir, and showed significant clinical improvement and return of lung function back to baseline. Currently, there is little evidence for using ganciclovir or valganciclovir for treatment of severe CMV infection in immunocompetent patients.

$\mathrm{CMV}$ is an unusual cause of pulmonary exacerbation in immunocompetent individuals with CF. It can present acutely and is heralded by lymphocytosis. Recognising the symptoms and clinical features of the infection is important to ensure appropriate management. Further studies are needed to assess the burden of CMV disease in $\mathrm{CF}$, reactivation of latent infection or re-infection, and to investigate the safety and efficacy of antiviral therapy in the immunocompetent adults.

Akhil Sawant ${ }^{1}$, Giulia Spoletini $\oplus^{1}$, Paul Whitaker ${ }^{1}$, Christine Etherington ${ }^{1}$, Ian Clifton $^{1}$ and Daniel Peckham (1) ${ }^{1,2}$

${ }^{1}$ Adult Cystic Fibrosis Unit, Leeds Teaching Hospitals NHS Trust, Leeds, UK. ${ }^{2}$ Leeds Institute of Biomedical and Clinical Sciences, Faculty of Medicine and Health, University of Leeds, Leeds, UK.

Correspondence: Daniel Peckham, Adult Cystic Fibrosis Unit, St James's University Hospital, Beckett Street, Leeds, UK. E-mail: d.g.peckham@leeds.ac.uk

Received: Sept 132017 | Accepted: Nov 172017

Conflict of interest: None declared. 


\section{References}

1 Flight WG, Bright-Thomas RJ, Tilston $\mathrm{P}$, et al. Incidence and clinical impact of respiratory viruses in adults with cystic fibrosis. Thorax 2014; 69: 247-253.

2 Etherington C, Naseer R, Conway SP, et al. The role of respiratory viruses in adult patients with cystic fibrosis receiving intravenous antibiotics for a pulmonary exacerbation. J Cyst Fibros 2014; 13: 49-55.

3 Barclay A, Naseer R, McGann H, et al. Cytomegalovirus pneumonia in an immunocompetent adult: a case report. Acute Med 2011; 10: 197-199.

$4 \mathrm{Yu}$ WL, Chen CM, Lee WY. Ventilator-associated cytomegalovirus organizing pneumonia in an immunocompetent critically ill patient. J Microbiol Immunol Infect 2017; 50: 120-122.

5 Cinel G, Pekcan S, Ozcelik U, et al. Cytomegalovirus infection in immunocompetent wheezy infants: the diagnostic value of CMV PCR in bronchoalveolar lavage fluid. J Clin Pharm Ther 2014; 39: 399-403.

6 Bosc C, Clement M, Deroux A, et al. Severe pneumonia due to cytomegalovirus in chronic obstructive pulmonary disease. Rev Mal Respir 2014; 31: 435-438.

7 Rawlinson WD, Boppana SB, Fowler KB, et al. Congenital cytomegalovirus infection in pregnancy and the neonate: consensus recommendations for prevention, diagnosis, and therapy. Lancet Infect Dis 2017; 17: e177-ee88.

8 Navarro D. Expanding role of cytomegalovirus as a human pathogen. J Med Virol 2016; 88: 1103-1112.

9 Ong EL, Ellis ME, Webb AK, et al. Infective respiratory exacerbations in young adults with cystic fibrosis: role of viruses and atypical microorganisms. Thorax 1989; 44: 739-742.

10 Pirson J, Etienne I, Ruiz-Patino M, et al. Cytomegalovirus (CMV) primoinfection caused serious deterioration in 2 young CF fathers. J Cystic Fibrosis 14: S141. 